\title{
Modelling of scour formation using SedFoam, continuum approach
}

\author{
Alaa Ghzayel ${ }^{1, *}$, Anthony Beaudoin ${ }^{1,}$, and Sebastien Jarny ${ }^{1,}$ \\ ${ }^{1}$ University of Poitiers, Institut PPrime, 86000 Poitiers, France.
}

\begin{abstract}
SedFOAM is a two-phase flow solver built upon consecutive laws, based on the CFD toolbox OpenFOAM. The sediment body is considered as a continuum phase. The intergranular and fluid stresses are modeled with the dense granular flow rheology and the $k-\epsilon$ turbulent model, respectively. The model setup will be based on an experimental study on the scour due to a water jet subjected to lateral confinement. A comparison study will be made based on precise experimental data by Martino et al. (2019) that will show the advantages and defaults of SedFoam. The objective of this work is to reproduce the digging and filling cycle of the scour formation due to the water jet in a confined canal. The first numerical results show that it needs to use 3D numerical simulations because of the fluctuation of the jet direction induced by the presence of a driven flow cavity.
\end{abstract}

\section{Introduction}

Two phase flow models for sediment transport have been widely developed in the past decades, in which dynamical equations are applied on both the fluid and particles phases where the particles are considered as a continuous body within the fluid. This approach enables us to take into consideration most of the physical processes such as particles interactions, turbulence modulation and particle interaction with another. Only very recently, a 3D simulation two- phase flow model, called SedFOAM, has been applied for sheet-flow sediment transport [1]. The intergranular and fluid stresses are modeled with the dense granular flow rheology [2] and the $k-\epsilon$ turbulent model [3]. In this work, we will use SedFOAM to simulate a case scenario of a hydro-sedimentary canal proposed by Martino et al. in 2019 [4]. Benchmarking will be done based on the experimental study of Martino et al. on the development of a scour and mound formation. For a certain flow rate, a digging and refilling cycle of the sediments inside the scour is observed. The objective of this work is to be able to reproduce numerically the same cycle of digging and refilling and to be able to conclude the phenomena behind this type of sediment erosion cycle. This numerical study will provide the advantages and defaults of SedFOAM.

\section{SedFoam}

\subsection{Mathematical approach}

To construct the model based on the Eulerian two-phase flow approach, the mass and momentum conservation equations have to be averaged over the fluid and particles phases. In order to be able to apply this formulation to turbulent flows, turbulence averaging is required. The mass conservation equations are as following:

*e-mail: Alaa.ghzayel@univ-poitiers.fr

A video is available at https://doi.org/10.48448/4cmw-wr92

$$
\frac{\partial \alpha}{\partial t}+\frac{\partial \alpha u_{i}^{a}}{\partial x_{i}}=0 \text { and } \frac{\partial \beta}{\partial t}+\frac{\partial \beta u_{i}^{b}}{\partial x_{i}}=0
$$

where $\alpha$ and $\beta(\beta=1-\alpha)$ are the particles and fluid volume concentrations, $u_{i}^{a}$ and $u_{i}^{b}$ are the particles and fluid velocities. The momentum equations for the particles and fluid phases are expressed as following:

$$
\begin{gathered}
\frac{\partial \rho^{a} \alpha u_{i}^{a}}{\partial t}+\frac{\partial \rho^{a} \alpha u_{i}^{a} u_{j}^{a}}{\partial x_{j}}=-\alpha \frac{\partial p}{\partial x i}+\alpha f i-\frac{\partial \tilde{p}^{a}}{\partial x i}+\frac{\partial \tau_{i j}^{a}}{\partial x j} \\
+\alpha \rho^{a} g_{i} \alpha f i+\alpha \beta K\left(u_{i}^{b}-u_{i}^{a}\right)-S_{U S} \beta K v_{t}^{b} \frac{\partial \alpha}{\partial x i}
\end{gathered}
$$

$$
\begin{aligned}
& \frac{\partial \rho^{b} \alpha u_{i}^{b}}{\partial t}+\frac{\partial \rho^{b} \alpha u_{i}^{b} u_{j}^{b}}{\partial x_{j}}=-\beta \frac{\partial p}{\partial x i}+\beta f i+\frac{\partial \tau_{i j}^{b}}{\partial x j} \\
& \quad+\beta \rho^{b} g_{i}+\beta f i-\alpha \beta K\left(u_{i}^{b}-u_{i}^{a}\right)-S_{U S} \beta K v_{t}^{b} \frac{\partial \alpha}{\partial x i}
\end{aligned}
$$

where $\rho^{a}$ and $\rho^{b}$ are the particles and fluid densities, $g_{i}$ is the gravitational acceleration, $f_{i}$ is an external force, $p$ is the pressure of the fluid, $\tau_{i j}^{b}$ is the fluid stress, $\tilde{p}^{a}$ and $\tau_{i j}^{a}$ are the particles normal and shear stresses, $k$ is the drag parameter, $v_{t}^{b}$ is the turbulent viscosity and the inverse of the Schmidt number $S_{U S}=1 / \sigma_{c}$. The $K-\epsilon$ model is implemented into the solver. It is a two equation model that gives a general description of turbulence by means of two transport equations that take into account the turbulent kinetic energy (TKE) and the rate of dissipation of the turbulent kinetic energy $(\epsilon)$.

\subsection{Model solution procedure}

The previous mathematical formulation is solved with the following procedure: 
1. Solve the sediment concentration $\alpha$ according to equation (1).

2. Update the concentration volume $\beta=1-\alpha$.

3. Calculate $K$, the drag parameter:

$$
K=0.75 C_{d} \frac{\rho^{b}}{d_{e f f}}\left\|u^{b}-u^{a}\right\| \beta^{-h_{E x p}}
$$

4. Solve the fluid turbulence parameter based on the chosen model and then calculate the eddy and fluid effective viscosities.

$$
\begin{gathered}
\frac{\partial k}{\partial t}+u_{j}^{b} \frac{\partial k}{\partial x_{j}}=\frac{R^{b t}{ }_{i j}^{j}}{\rho^{b}} \frac{\partial u_{i}^{b}}{\partial x_{j}}+\frac{\partial}{\partial x_{j}}\left[\left(v^{b}+\frac{v_{t}^{b}}{\sigma_{k}} \frac{\partial k}{\partial x_{j}}\right]-\epsilon\right. \\
-\frac{2 K\left(1-t_{m f}\right) \alpha K}{\rho^{b}}-\frac{S_{U S}}{\beta} v_{t}^{b} \frac{\partial \alpha}{\partial x_{j}}\left(\frac{\rho^{a}}{\rho^{b}}-1\right) g_{j}
\end{gathered}
$$

5. Solve the particles phase stress based on the chosen model, the dense granular-flow rheology:

$$
\tilde{\tau}_{i j}^{a}=\mu(I) \tilde{p}^{a} \frac{S_{i j}^{a}}{\sqrt{2 S_{i j}^{a} \cdot S_{i j}^{a}}}
$$

6. Solve the velocity-pressure coupling.

\section{Scour due to a water jet}

\subsection{Experimental setup}

SedFOAM is used to simulate the hydro-sedimentary canal setup used in the experimental study proposed by Martino et al.. The hydro-sedimentary canal setup is a narrow channel of dimensions $2 \mathrm{~cm}$ wide and $140 \mathrm{~cm}$ long, with a glass side walls and flow rate Q controlled by a pump. In the bottom of the canal, there is a flat granular layer of $10 \mathrm{~cm}$ depth and $60 \mathrm{~cm}$ length composed of glass beads of a median diameter of $1.1 \mathrm{~mm}$ and density of 2.65 $\mathrm{g} / \mathrm{cm}^{3}$. The water depth was $130 \mathrm{~mm}$. A sluice gate is located at the upstream of the system with an aperture $b_{0}$. A rotameter measures the flow rate $Q$ that can range from 2.5 l/min up to $17 \mathrm{l} / \mathrm{min}$. Thus the Reynolds number ranges between 3790 and 16200 . The benchmark experiment consists of an aperture $b_{0}=1 \mathrm{~cm}$ and $Q=6.5,7.5,8.5$ and $11.5 \mathrm{l} / \mathrm{min}$. The setup is shown in figure 1 .

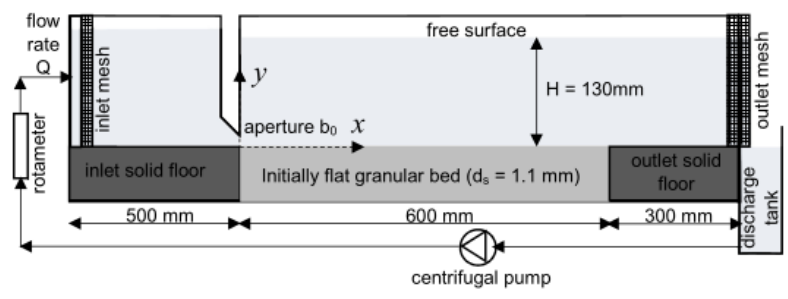

Figure 1: Experience setup by Martino et al. (2019).

\subsection{Dynamics of the scour process}

To understand the process, the scour formation submits three different phases (see Fig. 2). In the first phase, the particles are extracted and transported forming the hole and settled downstream to the right forming a mound which is a very quick process. This process barely took few seconds for the formation of the hole. In a development phase, the process slows down and the rate of particles transport decreases. The formation changes from a triangular shape to a trapizoidal shape, the more the particles accumulate the weaker the flow jet can transport particles which leads to accumulation of particles on the left side of the mound until the scour reaches a maximum depth and the grains on the left side of the mound migrate downslope. In a long term phase, after reaching a critical value of the resistance of the mound system and when the jets is not able anymore to support this mound, the left side of the mound collapses into the scour due to gravity in a steady recirculation regime and the hole will be partially refilled and this process will be repeated causing a digging and refilling cycles.

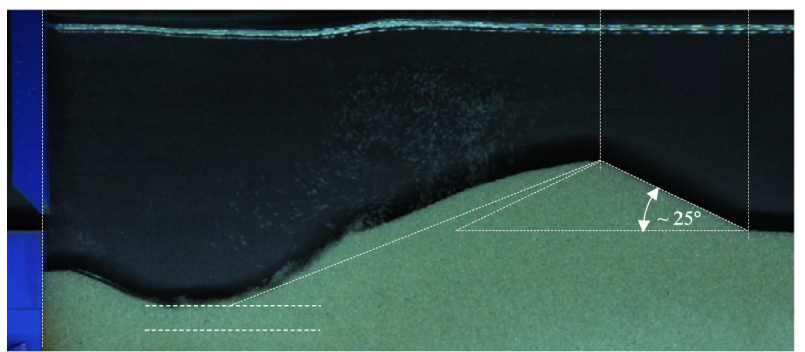

Figure 2: The digging-refilling (DR) regime, for $b_{0}=1 \mathrm{~cm}$ and $Q=10 \mathrm{l} / \mathrm{min}$.

\subsection{Criteria for comparison}

To analyse this process, we had to take into account the variables and compare them with constant values to check if there is a specific pattern. From this perspective, we consider the critical time $t_{c}$ where the mound submits a morphological change from a triangular to a trapizoidal shape as mentioned in the previous section. In the experiment done by Martino et al., $t_{c}$ varies from 8 to 15 seconds for the tested values of the flow rate $Q . t_{c}$ decreases with $Q$. Furthermore, an evolution of the maximum depth of the scour $Y_{0}$ with $Q$ should also be taken into account, to be able to understand the scour process in both the steady regime and the digging and refilling cycle. Figure 4 presents the time evolution of $Y_{0}$ for different value of $Q$. It has been observed that for a flow rate value lower than a certain critical flow rate, a transaction between the steady regime (SR) and the digging and refilling regime (DR) takes place. During the process, all the depths of the scour have deviations around the mean maximum value $Y_{0}$. The amplitude of deviations increases with the flow rate $Q$. 


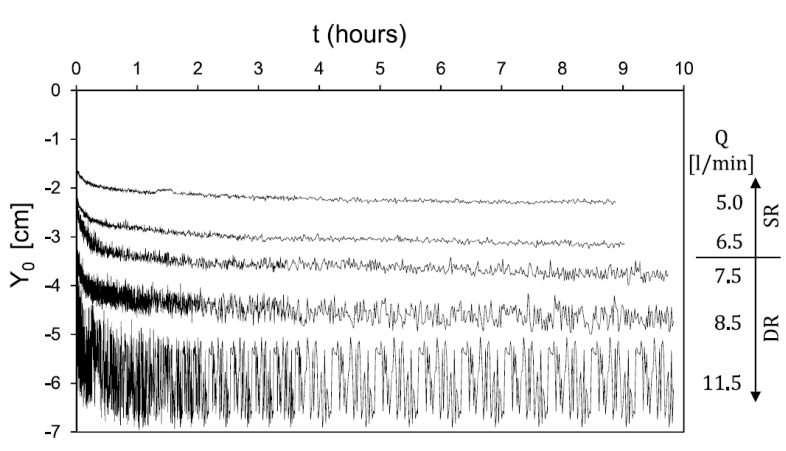

Figure 3: Time evolution of the maximum depth $Y_{0}$ for different flow rate $\mathrm{Q}$ and $b_{0}=1 \mathrm{~cm}, \mathrm{SR}$ is the steady regime and DR is the digging-refilling regime (Martino et al., 2019).

\section{Preliminary results}

\subsection{Numerical setup and Mesh construction}

The flow conditions and particles properties are set according to the experiment done by Martino et al.. The particle diameter is set to be $1.1 \mathrm{~mm}$ with a density of $2650 \mathrm{~kg} / \mathrm{m}^{3}$. In the dense granular flow rheology, the coefficients of friction are fixed to $\mu_{s}=0.4$ and $\mu_{2}=0.6$, as it has been proposed in the literature for glass beads. The water flow velocity profile was introduced at the inlet boundary condition using the equation proposed by Martino et al.:

$$
\begin{gathered}
\frac{U}{U m}=A_{1}\left(\frac{y}{y_{1 / 2}}\right)^{1 / 7}\left[1-\operatorname{erf}\left(A_{2} \frac{y}{y_{1 / 2}}\right)\right], \\
\frac{U_{m}}{U_{0}}=2\left(\frac{x}{b_{0}}\right)^{-1 / 3}
\end{gathered}
$$

where $U_{m}$ and $U_{o}$ are the maximum water jet velocity and the mean water velocity, $A_{1}$ and $A_{2}$ are non-dimensional constant fit parameters that are equal to 1.48 and 0.68 respectively and $y_{1 / 2}$ is the vertical position of the velocity where $U=U_{m} / 2$. The results of the numerical water velocity profile of both the 2D and 3D models are in good agreement with the proposed equation based on the measured experimental velocity profile (see Fig. 4).

The computational domain has the dimensions with $z=$ $-0.1 \mathrm{~m}$ and a water column of $z=0.15 \mathrm{~m}$ and a canal length of $x=0.3 \mathrm{~m}$. BlockMesh, a meshing utility that is part of extend-Foam in OpenFOAM, was used to construct the finite volume mesh. In 2D, the mesh is a quasi 3D mesh with only one layer in the third direction. The mesh is defined with a cell size in the $\mathrm{x}$-axis direction (stream-wise direction) of a value $\Delta_{x}=0.0012 \mathrm{~m}$ and in the vertical direction with a value of $\Delta_{y}=0.0005 \mathrm{~m}$ for the particles body and $\Delta_{y}=0.0023 \mathrm{~m}$ in the water column. In 3D, the same setup was used but the mesh was extended 20 cells in the third direction, making the cells number 20 times more than the $2 \mathrm{D}$ mesh. It will influence dramatically the CPU time. The CPU time will increase from few days in 2D to about 2 weeks in 3D for a real time simulated of 20 seconds. The time steps were controlled by the Courant number $C_{0}=0.1$ that was taken smaller than 1 to avoid numerical instability.

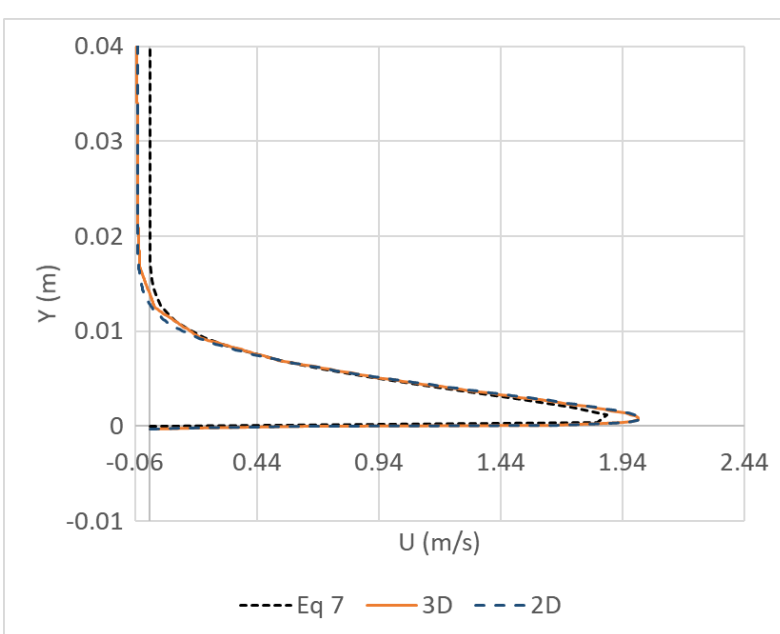

Figure 4: Measured experimental water velocity profile in comparison with the numerical velocity profiles for $Q=11.5 \mathrm{l} / \mathrm{min}$ in $2 \mathrm{D}$ and $3 \mathrm{D}$.

\subsection{D numerical simulations}

Four different flow rate $Q$ were used in the 2D numerical simulation on SedFOAM, $Q=6.5,7.5,8.5$ and $11.5 \mathrm{l} / \mathrm{min}$. The scour and dune formation were observed, but the dune stabilized with a triangular shape, no change in shape was observed (see Fig. 5).
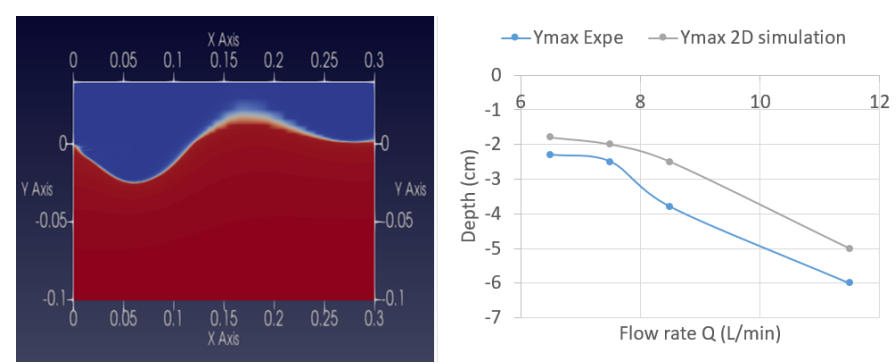

Figure 5: 2D numerical results of the scour and dune formation with $Q=8.5 \mathrm{l} / \mathrm{min}$ (right) and $Y_{\max }$ the maximum erosion depth for different flow rate $Q$ compared with the experimental data (right).

The maximum scour depth was recorded. As the experiment showed, it increases with increasing flow rate. The numerical and experimental results have approximately the same trend, but the scour depth was higher in the experiment as shown in figure 5. As for the water flow, the jet was always following the morphological change, and there was no fluctuation on the water. This behavior of water flow (see Fig. 6) is not in good agreement with the literature, thus a 3D simulation has to be made, to take into account the turbulent flow fluctuation inside the scour.

\subsection{D numerical simulations}

The 2D numerical simulations couldn't reproduce the fluctuation flow pattern caused by the scour and mound formation process observed in the experiment. Thus the 3D 

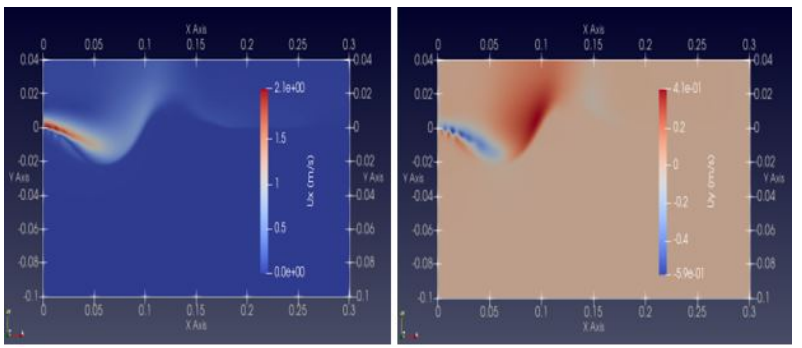

Figure 6: 2D numerical results of the water flow jet downstream of the sluice gate of aperture $b_{0}=1 \mathrm{~cm}$ (left: $U_{x}$ and right: $U_{y}$ ).

numerical simulations were more appropriate to solve this issue. The water flow in the $3 \mathrm{D}$ numerical simulations is described as following, at first, the water jet is flowing with the particle bed until the scour starts to form, at this point, a fluctuation of the flow in the scour will be observed with a backward re-circulation, that leads to move the jet slightly upward, at the same time, the dune will cause jet flipping towards the surface (see Fig 7). This description in the literature is also observed.
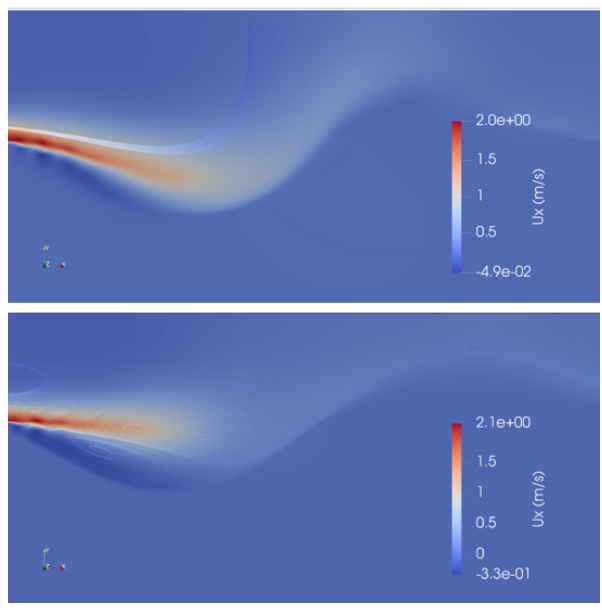

Figure 7: 3D numerical results of the water flow jet streamlines downstream of the sluice gate of aperture $b_{0}=1 \mathrm{~cm}$ with $Q=11.5 \mathrm{l} / \mathrm{min}$ at two times $t=1 \mathrm{~s}$ (top) and $t=10 \mathrm{~s}$ (bottom).

As for the scour and dune formation, a change in the shape of the dune was observed in the 3D numerical simulations (see Fig 8) unlike the 2D numerical simulations while the scour depth was less than the experimental measurements, which indicate that the model needs more optimisation and parametric study to be able to reproduce the experimental observations as accurate as possible.

\section{Conclusion and perspectives}

To be able to take into account the water flow fluctuation, a 3D multi phase flow model has to be used in the sense where the flow fluctuation pattern is not observed in simple 2D numerical simulations as well as the change of the form of the mound as shown in the previous sections. The phenomena of jet flipping and formation of a cavity reversed flow and change of the mound form was observed in the 3D numerical simulations. In contrast, the maximum depth in the 3D numerical simulations was far less than the experimental results while in the 2D simulations, the maximum scour depth was almost in good agreement with the numerical data. Furthermore, the cycle of digging and refiling was not observed in any of the numerical simulations and a difference of the scour depth between the $2 \mathrm{D}$ and $3 \mathrm{D}$ numerical simulation raise questions on the calibration of the numerical model's rheology parameters of the inter-granular stresses as well as the hydrodynamic of the model, as in the proposed equation only the $\mathrm{x}$-axis direction is considered and there is no enough data on the turbulence model used. This work is still a work in progress. More improved numerical simulations will be performed during the period of the study to assure the compatibility of the physical models parameters with the experimental data. To take into account the missing water velocities of the water flow in the other directions as well as the parameters of the turbulent model, interFoam, a hydrodynamic solver of OpenFoam, will be used to simulate the flow downstream of a sluice gate to be able to produce the missing parameters numerically and introduce them inside the SedFoam simulations instead of the water jet equation used previously.

\section{References}

[1] Z. Cheng, T.-J. Hsu, J, Chauchat, Adv. Water Res. 111, 205-223 (2018)

[2] GDRmidi, Eur. Phys. J. E 14, 341-365 (2004)

[3] R. Bakhtyar, A. Yeganeh-Bakhtiary, D. Barry, A. Ghaheri, Adv. Water Resour. 32, 1267-1283 (2009)

[4] Román G. Martino, Francisco García Ciani, Agnes Paterson, Marcelo F. Piva, European Journal of Mechanics / B Fluids 75, 219-227 (2019)

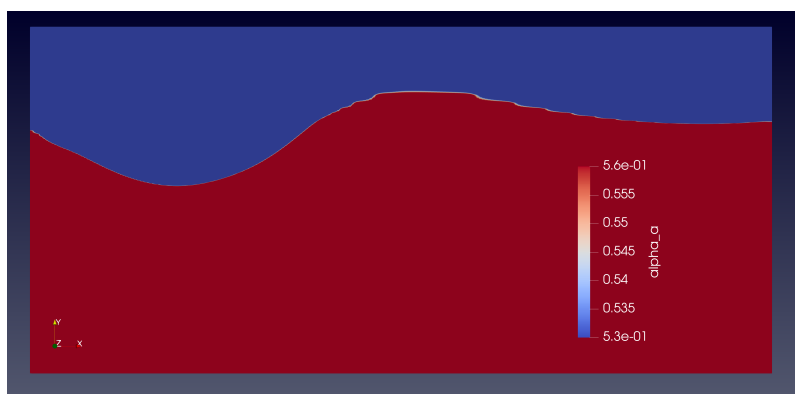

Figure 8: 3D numerical results of the scour and dune 